\title{
PRESENÇA DE FATORES DE RISCO PARA DOENÇAS CARDIOVASCULARES EM ADOLESCENTES
}

\author{
PRESENCE OF RISK FACTORS FOR CARDIOVASCULAR DISEASE IN ADOLESCENTS \\ PRESENCIA DE FACTORES DE RIESGO DE ENFERMEDADES CARDIOVASCULARES EN \\ ADOLESCENTES
}

José Alexandre do Nascimento ${ }^{1}$, Larisse Lobão de Sousa Lima², Maiara Naiara Mesquita de Lima ${ }^{3}$, Adriana Ponte Carneiro de Matos $^{4}$

\begin{abstract}
As doenças cardiovasculares são os principais motivos de morbimortalidade no mundo. Alguns fatores de risco adotados na fase da adolescência podem estar presentes até a vida adulta, aumentando a predisposição ao desenvolvimento dessas doenças na população. Identificar os fatores de risco para o desenvolvimento das doenças cardiovasculares em adolescentes. Uma pesquisa de caráter descritivo, transversal e exploratório, composto por uma amostra de 208 alunos. Foram avaliados os hábitos alimentares, estilo de vida, perfil sociodemográfico e perfil físico, por meio de questionário estruturado. Foram avaliados 208 adolescentes, sendo que o sexo masculino foi predominante (55\%); a média de idade foi de 16,37 $\pm 0,85$ anos. Os fatores mais evidentes foram o sedentarismo (51\%), seguido do consumo excessivo de alimentos ricos em sódio e gordura (79\%). Foram identificados fatores de risco para doenças cardiovasculares, como o sedentarismo, consumo excessivo de sódio (82\%), consumo de gordura (85\%) e ambos (79\%).
\end{abstract}

Palavras-Chave: Doenças Cardiovasculares; Saúde do Adolescente; Fatores de Risco.

\section{ABSTRACT}

Cardiovascular diseases are the main reasons for morbidity and mortality in world. Some risk factors adopted in adolescence may be present until adulthood, increasing the population's predisposition to the development of these diseases. Identify risk factors for the development of cardiovascular disease in adolescents. A descriptive, transversal and exploratory research, consisting of a sample of 208 students. Eating habits, lifestyle, sociodemographic profile and physical profile were evaluated through a structured questionnaire. 208 adolescents were evaluated, where the male sex was predominant $55 \%$, the average age was $16.37 \pm 0.85$ years. The most evident factors were sedentary lifestyle $51 \%$, followed by excessive consumption of foods rich in sodium and fat (79\%). Risk factors for cardiovascular disease were identified, such as sedentary lifestyle, excessive consumption of sodium (82\%) and fat (85\%) and both (79\%).

Keywords: Cardiovascular Diseases; Adolescent Health; Risk Factors.

\section{RESUMEN}

Las enfermedades cardiovasculares son las principales causas de morbilidad y mortalidad em mundo. Algunos factores de riesgo adoptados en la adolescencia pueden estar presentes hasta la edad adulta, aumentando la predisposición de la población al desarrollo de estas enfermedades. Identificar factores de riesgo para el desarrollo de enfermedad cardiovascular en adolescentes. Investigación descriptiva, transversal y exploratoria, conformada por uma muestra de 208 estudiantes. Se evaluaron hábitos alimentarios, estilo de vida, perfil sociodemográfico y físico mediante un cuestionario estructurado. Se evaluaron 208 adolescentes, donde predominó el sexo masculino 55\%, la edad promedio fue de 16,37 $\pm 0,85$ años. Los factores más evidentes fueron el sedentarismo $51 \%$, seguido del consumo excesivo de alimentos ricos en sodio y grasas (79\%). Se identificaron factores de riesgo de enfermedad cardiovascular, como el sedentarismo, el consumo excesivo de sodio (82\%) y grasas (85\%) y ambos (79\%).

Palabras Clave Enfermedades Cardiovasculares; Salud Adolescente; Factores de Riesgo.

\footnotetext{
${ }^{1}$ Escola de Saúde Pública do Ceará, Fortaleza, Brasil. (0000-0001-6671-5517)

${ }^{2}$ Centro Universitário Estácio do Ceará, Fortaleza, Brasil. (0000-0003-3465-6844)

${ }^{3}$ Centro Universitário Estácio do Ceará, Fortaleza, Brasil. (0000-0001-5121-0436)

${ }^{4}$ Universidade Estadual do Ceará, Fortaleza, Brasil. (0000-0002-8680-3853)
} 


\section{INTRODUÇÃO}

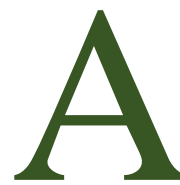

s doenças do aparelho cardiovascular são consideradas um dos principais motivos de morbimortalidade no planeta e sua incidência é crescente nos países em processo de desenvolvimento. Borges et al. ${ }^{1}$ chamam a atenção para um intenso choque ocasionado por esses problemas nas classes sociais menos beneficiadas, referenciando como a principal causa de óbitos, no Brasil, com uma média de $30 \%$ em algumas faixas de idades específicas. Dados da Organização Pan-Americana de Saúde (OPAS) ${ }^{2}$ mostraram que, no ano de 2015, uma estimativa de 17,7 milhões de pessoas vieram a óbito em decorrência das doenças cardiovasculares (DCV's), chegando a uma porcentagem de $31 \%$ a nível mundial. Cerca de $3 / 4$ dos óbitos da população atingiu países de média e baixa renda.

A Sociedade Brasileira de Cardiologia $(\mathrm{SBC})^{3}$, por meio do Cardiômetro, instrumento online que indica o número de óbitos causados pelas DCV's, no Brasil, calculou uma estimativa de 345.111 mil mortes, no ano de 2015. No ano de 2016, a estimativa chegou a 349.938 mil, comprovando a alta incidência que essas doenças vêm causando em nossa população. Segundo Eyken et al. ${ }^{4}, 80 \%$ dos óbitos causados por essas doenças estão associados a fatores de risco já presentes em nosso ambiente. De acordo com Ribeiro et al. ${ }^{5}$, os principais fatores de risco modificáveis nas doenças cardíacas são: os hábitos alimentares incorretos, a obesidade, o sedentarismo, o estilo de vida e o tabagismo.

No estudo comandado por Junior et $\mathrm{al}^{6}$., realizado com jovens de 14 a 17 anos que cursavam o ensino médio, foi constatado que $51,4 \%$ dos adolescentes avaliados possuíam mais de dois fatores de risco para tais doenças, concordando com dados do estudo de Brito et al. ${ }^{7}$, no qual $64,3 \%$ de adolescentes do sexo masculino e $50,6 \%$ do sexo feminino apresentaram peso acima do recomendado.
Ribas e Silva ${ }^{8}$ avaliaram 571 indivíduos, sendo que o sobrepeso, a dislipidemia, o sedentarismo e familiares que possuíam alguma doença cardiovascular foram os fatores de risco mais identificados. Cerca de $39 \%$ das crianças que foram avaliadas nesse estudo apresentaram três ou mais fatores de risco.

A partir disso, órgãos internacionais vêm preconizando programas que ofertem a promoção de saúde nas instituições de ensino, pois o período da adolescência é o momento apropriado para a formulação de estratégias de intervenção direcionadas ao combate das doenças cardíacas. Existem diversas evidências de que essas doenças se originam neste período da vida. Convém lembrar ainda que alguns fatores de risco adotados na fase da adolescência podem estar presentes até a vida adulta, aumentando a predisposição ao desenvolvimento dessas doenças na população?

Embora haja diversos estudos e vários investimentos direcionados ao controle dessas doenças, os números de mortes e incapacidades sofreram poucas mudanças. Os programas que visam à promoção de saúde e mudanças no estilo de vida que trazem prejuízo à saúde são os que mais trazem resultados satisfatórios ${ }^{1}$.

Os serviços ofertados pela Atenção Primária à Saúde (APS) são importantes para os sistemas que integram a saúde, pois é recorrente a alta prevalência das doenças cardíacas em nossa sociedade. Sem esses serviços, pacientes acometidos por doenças do aparelho circulatório estão mais tendentes à hospitalização, levando a uma debilidade em suas funções corporais e, através disso, adquirindo comorbidades ${ }^{10}$.

Atrelado a esse contexto, o Ministério da Educação (MEC) implementou o Programa Saúde nas Escolas (PSE) ${ }^{11}$, o qual busca uma relação permanente entre as instituições de educação e saúde, ofertando uma melhor qualidade de vida a nossa população. O objetivo do programa é fornecer um reforço para uma capacitação de forma integral dos alunos, por meio de ações que ofereçam promoção, prevenção e atenção à saúde, 
encarando os problemas que afetam o desenvolvimento dos alunos da rede pública de ensino.

Para alcançar uma eficácia positiva nessas ações, a escola se mostra um local apropriado para essa prática, visando o progresso da qualidade de saúde e condição nutricional. É um âmbito propício para a consolidação de iniciativas de promoção em saúde, ofertando apoio ao desenvolvimento humano e relações harmoniosas que contribuam para seu crescimento ${ }^{12}$.

Diante do exposto, o presente estudo teve como objetivo verificar em uma escola pública estadual, na cidade de Fortaleza, qual a frequência de adolescentes na faixa etária de 14 a 19 anos que apresentam fatores de risco para as DCV's. Uma vez identificados tais fatores na adolescência, é possível implementar ações de promoção e prevenção de saúde, revertendo esse cenário antes que ocasione comprometimentos mais complexos à saúde desses indivíduos.

\section{METODOLOGIA}

Tratou-se de uma pesquisa de caráter exploratório, transversal, descritivo de abordagem quantitativa, constituída por adolescentes escolares do ensino médio, sendo realizada em uma Escola de Ensino Médio de Tempo Integral (EEMTI), situada na cidade de Fortaleza- CE, no período de março a junho de 2019.

A população foi composta por 440 alunos, sendo a amostra igual a 208 alunos, baseada em cálculo para amostra finita. Os critérios de inclusão aplicados foram: alunos matriculados regularmente na escola, que tivessem o Termo de Consentimento Livre e Esclarecido (TCLE) e o Termo de Assentimento (TA) assinado pelos pais e alunos; possuir idade entre 14 e 19 anos; de ambos os sexos. Foram excluídos da pesquisa os alunos que possuíam alguma doença cardiovascular préexistente, diabetes mellitus e que não receberam autorização dos pais para participar do estudo.

Os dados do estudo foram coletados a partir de uma avaliação por meio de exame físico e entrevista, realizada em uma sala reservada da instituição escolar. A entrevista foi aplicada através de um questionário elaborado pelos próprios autores, contendo informações sociodemográficas (nome, idade, sexo), hábitos de vida (nível de prática de atividade física), hábitos alimentares (consumo de alimentos ricos em sódio e gordura), doenças pré-existentes na família (Diabetes, Hipertensão Arterial Sistêmica, Acidente Vascular Encefálico, Infarto Agudo do Miocárdio) e informações referentes às características físicas dos indivíduos, como altura, peso, circunferência abdominal e Índice de Massa Corpórea, que seguiu a Diretriz Brasileira de Diagnostico e Tratamento da Síndrome Metabólica ${ }^{19}$. Também foram mensuradas a glicemia e a pressão arterial. A mensuração das variáveis mencionadas foram todas obtidas pelos pesquisadores.

Para quantificar a pressão arterial dos alunos, foi utilizado um esfigmomanômetro e estetoscópio da marca Premium ${ }^{\circledR}$, e as etapas da aferição seguiram as recomendações da $6^{\circ}$ Diretriz de Monitorização Ambulatorial da Pressão Arterial $^{20}$. Já para quantificar o peso dos alunos, utilizamos uma balança digital da marca Livstar®. A glicemia foi obtida a partir de um glicosímetro fabricado pela Accu-Chek®, seguindo as orientações das Diretrizes da Sociedade Brasileira de Diabetes ${ }^{21}$.

Para uma maior comodidade e preservação dos alunos em relação as suas informações pessoais, a entrevista ocorreu de forma individual, em uma sala fechada. Para não haver divergências nas mensurações, a pressão arterial, a glicemia, o peso e a obtenção das medidas da circunferência abdominal e altura foram realizadas pelo mesmo pesquisador.

Para quantificar o peso e altura, foi solicitado aos alunos que retirassem o tênis, se posicionassem no centro da balança e ficassem de cabeça erguida, olhando para um ponto marcado na parede. Já em relação à circunferência abdominal, solicitamos que os mesmos levantassem a blusa até a região superior à cicatriz umbilical, para não 
haver uma falsa medida. As variáveis obtidas a partir da coleta de dados foram trabalhadas a partir da análise descritiva, por meio de médias e desvio padrão e frequências através de porcentagem. $\mathrm{O}$ tratamento das estatísticas foi realizado por meio do software Microsoft Office Excel versão 2016.

O presente estudo foi aprovado pelo Comitê de Ética e Pesquisa (CEP) do Centro Universitário Estácio do Ceará, sob Protocolo CEP n ${ }^{\circ}$ 2.801.273, estando de acordo com orientações para pesquisas envolvendo seres humanos, respaldado pela resolução no 510/2016, do Conselho Nacional de Saúde (CNS).

\section{RESULTADOS}

Foram avaliados 208 adolescentes, sendo 114 (55\%) do sexo masculino e 94 (45\%) do sexo

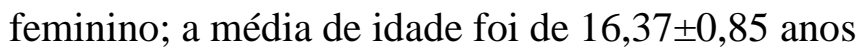
e a mediana igual a 16 anos. A renda familiar está descrita a partir da média, sendo 1,79 $\pm 0,93$ salários. Na Tabela 1 estão descritos os dados da caracterização demográfica dos indivíduos participantes do estudo.

Tabela 1 - Características demográficas dos indivíduos: sexo e etnia.

\begin{tabular}{ccc}
$\begin{array}{c}\text { Características } \\
\text { demográficas }\end{array}$ & Número de indivíduos & Total (\%) \\
\hline \hline Sexo & 114 & \\
M & 94 & $55 \%$ \\
F & & $45 \%$ \\
Etnia & 23 & \\
N & 30 & $11 \%$ \\
B & 32 & $14 \%$ \\
A & 123 & $59 \%$ \\
P & & \\
\hline
\end{tabular}

Fonte - Dados da Pesquisa.

$O$ perfil da amostra estudada foi caracterizado por uma população jovem, com predominância do sexo masculino. Além disso, evidenciamos que grande parte da amostra estudada morava exclusivamente com a mãe, sendo o grau mais prevalente de escolaridade das mães o ensino médio concluído (30\%) e não haviam concluído o ensino fundamental (24\%).
Tabela 2 - Distribuição de prevalência: etilismo, tabagismo, sedentarismo, consumo excessivo de sódio e consumo excessivo de alimentos gordurosos.

\begin{tabular}{cc}
\hline Variáveis & n (\%) \\
\hline \hline Etilismo & $52(25 \%)$ \\
Tabagismo & $10(5 \%)$ \\
Sedentarismo & $107(51 \%)$ \\
Consumo excessivo de alimentos com sódio & $170(82 \%)$ \\
Consumo excessivo de alimentos gordurosos & $176(85 \%)$ \\
\hline onte - Dados da Pesquisa.
\end{tabular}

Na Tabela 2 estão descritos os dados referentes à prevalência de sedentarismo, etilismo, tabagismo, consumo excessivo de sódio e consumo excessivo de alimentos gordurosos. Dados desse estudo demonstraram que 107 indivíduos (51\%) eram sedentários. Em seguida, na Tabela 3, estão dispostos os perfis antropométricos dos alunos, referentes à média de altura, peso, circunferência abdominal (CA) e o Índice de Massa Corpórea (IMC).

Levando em consideração o uso associado do álcool e o tabaco, identificamos que 9 estudantes $(4,37 \%)$ associavam esses dois fatores. Apesar de haver um baixo percentual, por se tratar de uma instituição de educação, isso pode levar a impactos negativos nos outros alunos.

Tabela 3 - Dados antropométricos dos alunos, referente ao IMC, CA, altura e peso.

\begin{tabular}{cc}
\hline Variáveis & $\mathbf{m} \pm \mathbf{d p}$ \\
IMC & $21,98 \pm 4,81$ \\
CA & $80,90 \pm 11,14$ \\
Altura & $1,65 \pm 0,09$ \\
Peso & $60,96 \pm 13,26$ \\
\hline FIMC: índice de massa corpórea; CA: circunferência abdominal; \\
Fonte - Dados da Pesquisa.
\end{tabular}

A partir do cruzamento dos dados consumo excessivo de alimentos ricos em sódio e gordura, identificamos que 163 (79\%) alunos avaliados ingeriam alimentos ricos tanto em sódio como em gordura. Quando perguntados se sentiam dor no peito ou cansaço após a realização de atividade física, $103(49,51 \%)$ referiram que sim.

Não identificamos a prevalência de HAS na amostra estudada. Os valores dos níveis pressóricos expressos a partir da média foram Pressão Arterial

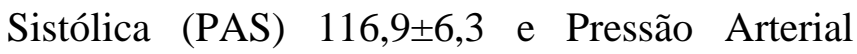
Diastólica (PAD) 90 $\pm 7,6$. 
Tabela 4 - Dados antropométricos dos alunos, referente ao IMC, CA, altura e peso.

\begin{tabular}{cc}
\hline Variáveis & Número de indivíduos (\%) \\
\hline \hline HAS & $99(48 \%)$ \\
IAM & $42(20 \%)$ \\
DM & $105(50 \%)$ \\
AVE & $53(25 \%)$ \\
\hline
\end{tabular}

*valores descritos a partir de porcentagem. Fonte - Dados da Pesquisa.

Tabela 5 - Dados antropométricos dos alunos, referente ao IMC, CA, altura e peso.

\begin{tabular}{ccc}
\hline IMC & $\mathbf{m} \pm \mathbf{d p}$ & $\%$ \\
\hline \hline Sexo feminino & & \\
$>16-24,9 \mathrm{~kg} / \mathrm{m}^{2}$ & $20,2 \pm 2,4$ & $32 \%$ \\
$25-39,9 \mathrm{~kg} / \mathrm{m}^{2}$ & $29,2 \pm 3,6$ & $13,6 \%$ \\
Sexo masculino & & \\
$>16-24,9 \mathrm{~kg} / \mathrm{m}^{2}$ & $19,5 \pm 2,3$ & $45,6 \%$ \\
$25-39,9 \mathrm{~kg} / \mathrm{m}^{2}$ & $30,4 \pm 2,4$ & $8,8 \%$ \\
\hline
\end{tabular}

Fonte - Dados da Pesquisa.

Na Tabela 4 estão descritos os dados relativos ao histórico familiar de HAS, DM, IAM e AVE. Na Tabela 5 descrevemos com mais detalhes os dados obtidos a partir do IMC, abordando separadamente o sexo feminino e o masculino. Os valores estão descritos a partir da média.

O uso de contraceptivos foi outra variável estudada, visto sua relação com o desenvolvimento das doenças cardiovasculares. A partir disso, 14 indivíduos $(14,9 \%)$ da amostra referiram fazer uso de contraceptivos. Realizamos também o cruzamento do uso de contraceptivos e o uso de tabaco, visto que a associação desses dois fatores aumenta a probabilidade de desenvolvimento do risco cardiovascular. Contudo, 3 estudantes $(3,19 \%)$ do público feminino avaliado consumiam tabaco e utilizavam contraceptivos.

\section{DISCUSSÃO}

Essa pesquisa experimentou algumas limitações em relação à obtenção dos dados. A mensuração da pressão arterial em um único momento não nos fornece bases para obtenção de valores fidedignos, levando em consideração a HAS, principal fator de risco para as doenças cardiovasculares. Outro fator limitante foi o conhecimento dos alunos acerca das informações familiares, como salário e comorbidades.

Nesse estudo podemos identificar uma alta prevalência em alguns fatores de risco cardiovasculares entre alunos adolescentes, como o sedentarismo. Além desses fatores, identificamos também hábitos alimentares irregulares, como o consumo excessivo de alimentos ricos em sódio e gordura.

Alguns estudos apontam que a relação da mãe com o adolescente é o fator de maior influência na formação dos hábitos alimentares, pois dentro do convívio familiar, mãe e filho dividem as mesmas condições socioeconômicas, estilo de vida e hábitos alimentares, interferindo em sua alimentação e na disponibilidade de alimentos ${ }^{1}$. Tal fato vai de encontro ao que identificamos em nossa pesquisa, na qual grande parte da amostra referiu conviver apenas com a mãe.

Outro achado importante está relacionado à baixa renda e à baixa escolaridade das mães dos alunos avaliados. $\mathrm{O}$ valor da renda familiar possui relação direta com o nível de escolaridade, interferindo diretamente sobre as oportunidades de empregos e salários. Logo, os baixos salários trazem impactos negativos sobre os hábitos alimentares e o estilo de vida que a família leva, pois limita a qualidade e quantidade do alimento obtido a partir da baixa renda ${ }^{13}$.

A cor parda foi a mais identificada em nosso estudo, seguida de indivíduos que referiam a cor amarela. Esse achado corrobora com Amorim et $\mathrm{al}^{14}$., que, estudando fatores de risco em adolescentes, identificou que os indivíduos com a cor parda constituíram $54,9 \%$ da amostra. Para Silva ${ }^{15}$, os indivíduos com cor/raça negra ao que 
parece apresentam um potencial fator hereditário relacionado a captação na célula de sódio e cálcio, onde a célula passa por um maior influxo de sódio e maior efluxo de cálcio, favorecendo o desenvolvimento da HAS. No entanto, a população de raça negra, foi a menos encontrada em nosso estudo.

O sedentarismo foi um dos fatores mais prevalente na população estudada. Junior et $\mathrm{al}^{16}$. encontraram resultados semelhantes, nos quais o nível de sedentarismo entre os adolescentes foi de $59,5 \%$. O nível de atividade física traz impactos diretos sobre o condicionamento muscular cardíaco e está diretamente relacionado às doenças crônicas.

Hábitos alimentares incorretos foram os fatores mais evidentes em nosso estudo, como o consumo excessivo de alimentos ricos em sódio e gordura. Esses dados confirmam os resultados de Beck et $\mathrm{al}^{17}$, nos quais $95,4 \%$ dos indivíduos consumiam alimentos ricos em ácidos graxos saturados e 59,4\% ricos em sódio.

Por fim, os dados pertinentes ao peso dos alunos não expressaram valores elevados, repercutindo de forma positiva no IMC desses indivíduos. Rodrigues et $\mathrm{al}^{18}$, ao final de seu estudo, obtiveram dados semelhantes aos encontrados nesta pesquisa, em que a média de peso foi de $50,75 \pm 14,95$.

Baseado nesses dados, é necessária a realização de ações de educação em saúde, como também uma regulamentação das cantinas nas escolas, adaptando seu cardápio para uma dieta mais nutritiva e saudável. Outra alternativa positiva seria implementar protocolos de avalição de saúde dos alunos, programas de prevenção de doenças, bem como oficinas que abordassem temáticas relevantes, levando a uma conscientização dos problemas de saúde que podem se desenvolver a partir de tais hábitos irregulares.

\section{CONSIDERAÇÕES FINAIS}

É evidente a presença de fatores de risco para as doenças cardiovasculares na população adolescente. Grande parte da amostra desse estudo experimenta ao menos dois fatores de risco cardiovascular e mais da metade apresentam três fatores. O sedentarismo e os hábitos alimentares incorretos foram os mais prevalentes. Diversos são os fatores que geram impacto sobre esses fatores, influenciando de forma negativa no hábito de vida saudável.

Portanto, torna-se fundamental uma avaliação minuciosa desses fatores e seus agentes etiológicos nessa população, atribuindo a esta avaliação práticas de promoção e prevenção em saúde, buscando resgatar a diminuição nos índices destes fatores. Ter hábitos saudáveis nessa fase da vida é primordial para uma boa saúde cardiovascular no futuro.

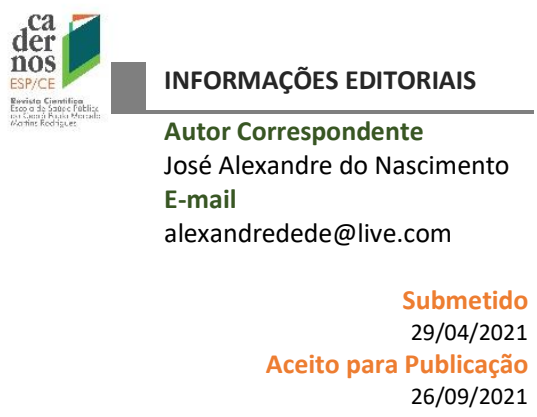




\section{REFERÊNCIAS}

1. Borges C, Busnello F, Pellanda L. Identificação de fatores de risco cardiovascular em pais/cuidadores de crianças cardiopatas. Arq Bras de Cardiologia. 2012;99(4):936-943.

2. Organização Pan-Americana de Saúde. [online]. Washington, Estados Unidos da América; 2017. Disponível em: http://www.paho.org/bra/index.php?option=com_content\&view=article\&id=5253:doencas cardiovasculares\&Itemid=839.

3. Sociedade Brasileira de Cardiologia [online]. Rio de Janeiro, Brasil; 2015. Disponível em: ttp://www.cardiometro.com.br/anteriores.asp.

4. Eyken E, Moraes C. Prevalência de fatores de risco para doenças cardiovasculares entre homens de uma população urbana do Sudeste do Brasil. Cad Saúde Pública. 2009;25(1):111-123.

5. Ribeiro A, Cotta R, Ribeiro S. A promoção da saúde e a prevenção integrada dos fatores de risco para doenças cardiovasculares. Ciência \& Saúde Col. 2012;17(1):7-17.

6. Júnior FJ, Mendes J, Barbosa D, Lopes A. Fatores de risco cardiovascular em adolescentes: prevalência e associação com fatores sociodemográficos. Rev Bras Epidemiologia. 2011;14(1):50-62.

7. Brito BB, Leal JDV, Formiga LMF, Frota KMG, Silva ARV, Lima LHO. Doenças cardiovasculares: fatores de risco em adolescentes. Revista Cogitare Enferm. 2016; 21 (2): 01-8.

8. Ribas SA, Silva LCS. Fatores de risco cardiovascular e fatores associados em escolares do Município de Belém, Pará, Brasil. Cad Saúde Pública. 2014;30(3): 577-86.

9. Romanzini M, Reichert F, Lopes A, Petroski É, Farias Júnior J. Prevalência de fatores de risco cardiovascular em adolescentes. Cad Saúde Pública. 2008;24(11):2573-81.

10. Lentsck M, Mathias T. Hospitalizations for cardiovascular diseases and the coverage by the family health strategy. Rev LatinoAmericana Enfer. 2015;23(4):611-9.

11. Ministério da Educação [online]. Brasília: MEC; 2018. Disponível em: http://portal.mec.gov.br/expansao-da-rede-federal/194secretarias-112877938/secad-educacao-continuada-223369541/14578-programa-saude-nas-escolas.

12. Schmitz B, Recine E, Cardoso G, Silva J, Amorim N, Bernardon R, et al. A escola promovendo hábitos alimentares saudáveis: uma proposta metodológica de capacitação para educadores e donos de cantina escolar. Cad Saúde Pública. 2008;24(2):312-22.

13. Sociedade Brasileira de Cardiologia. Diretriz Sul-Americana de prevenção e reabilitação cardiovascular. Arq Brasileiros Cardiologia. 2014; 103(2): 1-31.

14. Oliveira M, Fagundes R, Moreira E, Trindade E, Carvalho T. Relação de indicadores antropométricos com fatores de risco para doença cardiovascular. Arq Brasileiros Cardiologia. 2010;94(4):478-485.

15. Gomes EB, Moreira TMM, Pereira HCV, Sales IB, Lima FET, Freitas CHA, Rodrigues DP. Fatores de risco cardiovascular em adultos jovens de um município do Nordeste brasileiro. Revista Bras Enferm. 2012;65(64):594-600.

16. Carnelosso ML, Barbosa MA, Porto CC. Prevalência de fatores de risco para doenças cardiovasculares na região leste de Goiânia (GO). Rev Ciência Saúde Col. 2010; 15(1): 1073-80.

17. Ferreira SD, Carballo FP, Sousa FF, Silva DMR. Prevalência e fatores associados ao sobrepeso/obesidade em crianças da rede privada de ensino de Divinópolis/MG.Cad Saúde Col. 2015; 23(3): 289-197.

18. Neto ODA, Silva RCR, Assis AMO, Pinto EJ. Fatores associados à dislipidemia em crianças e adolescentes de escolas públicas de Salvador, Bahia. Revista Bras Epidem. 2012; 15(2): 335-45.

19. Sociedade Brasileira de Cardiologia. I Diretriz Brasileira de Diagnóstico e Tratamento da Síndrome Metabólica. Arq Bras de Card. 2005; 80(s.1): 1-28.

20. Sociedade Brasileira de Cardiologia. $6^{\circ}$ Diretrizes de Monitorização Ambulatorial da Pressão Arterial. Arq Bras de Card. 2018; 110(5): 1-29.

21. Sociedade Brasileira de Diabetes. Diretrizes da Sociedade Brasileira de Diabetes 2019-2020. Brasil. Disponível em: https://www.google.com/url?sa=t\&rct=j\&q=\&esrc=s\&source=web\&cd=\&ved=2ahUKEwiditaurL7yAhVxIrkGHTmmAN0QFn oECAIQAQ\&url=http\%3A\%2F\%2Fwww.saude.ba.gov.br\%2Fwp-content\%2Fuploads\%2F2020\%2F02\%2FDiretrizesSociedade-Brasileira-de-Diabetes-2019-2020.pdf\&usg=AOvVaw1NMMVYUeMDDr598RbBEvG3. 